De quelques enjeux et usages historiques du Français fondamental

\title{
La grammaire du Français fondamental: Interrogations historiques et didactiques
}

Dominique Klinger et Georges Daniel Véronique

\section{(2) OpenEdition Journals}

Édition électronique

URL : https://journals.openedition.org/dhfles/1189

DOI : $10.4000 /$ dhfles. 1189

ISSN : 2221-4038

Éditeur

Société Internationale pour l'Histoire du Français Langue Étrangère ou Seconde

Édition imprimée

Date de publication : 1 janvier 2006

ISSN : 0992-7654

Référence électronique

Dominique Klinger et Georges Daniel Véronique, « La grammaire du Français fondamental:

Interrogations historiques et didactiques ", Documents pour I'histoire du français langue étrangère ou seconde [En ligne], 36 | 2006, mis en ligne le 19 novembre 2014, consulté le 27 mai 2021. URL : http:// journals.openedition.org/dhfles/1189; DOI : https://doi.org/10.4000/dhfles.1189

Ce document a été généré automatiquement le 27 mai 2021

(c) SIHFLES 


\title{
La grammaire du Français fondamental: Interrogations historiques et didactiques
}

\author{
Dominique Klinger et Georges Daniel Véronique
}

\section{Introduction}

1 Cette contribution réfléchit aux conditions de construction d'une grammaire dans le cadre de l'entreprise du Français élémentaire, ultérieurement rebaptisé « fondamental ", et à ses prolongements dans les Constructions fondamentales du français (Le Goffic et Combe Mc Bride 1975). Notre travail cherche à préciser l'observation de J. C. Chevalier (1984 : 123) selon laquelle « il est curieux de constater la part réduite de la syntaxe dans le Français élémentaire, malgré la compétence dans le domaine de G. Gougenheim et la présence de Benveniste à la tête d'une commission Grammaire ».

2 En effet, si l'entreprise du Français fondamental ne parvient pas véritablement à formuler une grammaire, ce travail sera repris ultérieurement, sans que cette tentative, à son tour, n'ait de suite.

3 La première partie de cet exposé est consacrée au traitement du verbe dans le Français fondamental 1er et 2ème degrés. Nous abordons ensuite les prolongements qu'en propose la grammaire de P. Le Goffic et de N. Combe Mc Bride, influencée par la théorie de M. Gross. 


\section{Le Français fondamental : des listes et des prescriptions}

\subsection{Les principes de base}

Le Français fondamental a été créé à partir de listes de fréquence de mots dans un corpus de français parlé et d'enquêtes sur la disponibilité de certains mots par rapport à une liste de centres d'intérêt. Le tout a fait l'objet d'une synthèse fondée sur un critère d'« empirisme rationnel » (cf. Gougengheim et al. 1956 / 1964). Ce n'est « ni un dictionnaire ni une grammaire $»^{1}$; il est conçu pour l'enseignement du français à des adultes ou à des enfants sans être directement utilisable par des apprenants. Ce n'est pas non plus une méthode d'enseignement. Les principes appliqués à l'élaboration du Français fondamental 1er et 2ème degré, se résument ainsi : articuler les parties "vocabulaire " et " grammaire » sans tendre à l'exhaustivité, le tout en n'adoptant ni doctrine, ni progression ${ }^{2}$. Le répertoire lexical constitué comprend donc une partie " grammaire " sous la forme de "mots grammaticaux », lesquels figurent parmi les unités de langue les plus fréquentes en discours. Cette grammaire se présente sous la forme de prescriptions, étayées par des dépouillements et des dénombrements spécifiques, analogues à ceux menés pour la liste de fréquence générale.

\subsection{Dépouillements et dénombrements grammaticaux}

5 Comme l'indiquent Gougenheim et al. (1956 / 1964 : 211), la grammaire du Français fondamental 1er degré et celle du 2ème degré résultent de dépouillements grammaticaux, d'une enquête particulière sur l'interrogation directe et indirecte, de relevés des fréquences verbales et de la liste générale de fréquence du français parlé. L'enquête grammaticale a porté essentiellement sur le syntagme verbal comme vecteur de la temporalité (expression du futur et du passé, dénombrements des plus-queparfaits de l'indicatif, des conditionnels présents et passés, et étude du subjonctif), sur les verbes introduisant le discours direct et indirect, et par là sur les complétives. Les phrases complexes ont également été étudiées à travers les enquêtes sur le subjonctif, le conditionnel, le gérondif et le participe présent.

De l'ensemble de ces données sont issues cent cinquante-deux prescriptions pour le premier degré et le deuxième degré respectivement, portant sur toutes les parties du discours, leur morphologie et leur syntaxe. Par exemple : «on n'enseignera que les formes qui sujet [...] et que objet [...], on enseignera dont; on exclura les constructions en de qui, duquel [...]» etc. Il en est de même pour le verbe. Ces prescriptions s'accordent avec l'option prise au départ d'un choix de mots basé sur la réalité de l'usage. L'idée maitresse est que la grammaire ne saurait être enseignée coupée de sa relation avec le lexique. Enfin, il existe un rapport de suite, sans qu'on puisse parler de progression, entre le Français fondamental 1er degré et le 2ème degré, d'où l'exclusion de certaines constructions du premier degré.

\subsection{Les parties du discours et les espèces de mots}

7 Malgré la constitution de listes, l'ordre des parties du discours et la nomenclature de la grammaire scolaire sont conservés. Dans la liste générale du Français fondamental, 
certains mots sont suivis d'un astérisque indiquant qu'ils figurent aussi dans une autre liste, ou dans celle dite de "mots spéciaux ", où sont incorporés les "mots grammaticaux ».

Parmi les "mots spéciaux », on trouve les noms de mois et de jours de la semaine, des déictiques (aujourd'hui, demain, hier etc.), et bien d'autres mots classés par étiquetage sémantique (" parenté », « quantité » etc.).

Cependant, aucun critère définitoire du " mot » n'est donné qui permette d'établir la différence entre mot "grammatical » et « non grammatical ». Ainsi des unités sont introduites en tant que « locutions conjonctives / adverbiales » (suivies de que) dans le Français fondamental 2ème degré. Ceci s'explique du fait que le subjonctif est également introduit dans le Français fondamental 2ème degré (ce point est abordé plus bas). Or, le subjonctif est une servitude de certaines de ces locutions qui peuvent apparaitre seules ou suivies de que (avant, après, depuis, ainsi). Ce n'est certes pas le cas de afin qui apparaît dans afin de glosé par afin que dans le Français fondamental 2éme degré, non accompagné d'astérisque. Doit-on comprendre qu'on a affaire à un mot lexical et non à un mot grammatical ? Est-ce parce qu'afin que / de est paraphrasable par d'autres locutions en que / de comme pour que, à dessein de, de /en sorte que etc. ? Ceci n'est certes pas le cas pour avant que / de. L'Élaboration du Français fondamental (p. 222) fournit une précision à propos de la prescription 103 : il est préconisé de laisser de côté afin que et afin de, comme étant purement littéraires. Ceci est justifié par le fait qu' "afin que ne figure dans aucun [des] textes » et qu' "afin de a été relevé une seule fois ». Ces unités font néanmoins l'objet d'une entrée dans la liste des mots sans qu'elles soient accompagnées d'astérisques; or on peut légitimement les considérer comme des mots grammaticaux.

\subsection{Du Français fondamental 1er degré au 2ème degré}

10 Le Français fondamental 2eme degré prend la suite du Français fondamental 1er degré sous la même forme. Leur articulation repose sur la différence faite entre " connaissances actives " et " connaissances passives " (Le Français fondamental 2ème degré, p.47, brochure 212 de l'INRP). Les premières sont celles que l'on acquiert et qu'il faudra pouvoir manier, par exemple, dans la conversation ; elles font essentiellement l'objet du Français fondamental 1er degré. Les deuxièmes sont celles qu'il faudra reconnaître et qui pourront être utiles pour la lecture ; elles sont davantage présentes dans le Français fondamental 2ème degré. L'introduction d'éléments nouveaux dans le deuxième degré, « suite » du premier degré, et la distinction de types de " connaissances ", supposent un enseignement progressif où la maîtrise de certains éléments est nécessaire avant d'en aborder d'autres.

\subsection{Le verbe dans le Français fondamental 1er et 2ème degrés}

\subsubsection{Le Français fondamental 1er degré}

11 Les formes verbales et leurs valeurs temporelles ont fait l'objet de dépouillements et de comptages dans le corpus du Français fondamental. Des verbes (non dotés d'astérisques) sont insérés dans la partie lexicale du Français fondamental 1er et 2ème degrés, dans la liste générale des mots retenus, alors que des prescriptions sont données dans la partie grammaticale sans qu'elles portent sur tous les verbes retenus. Ils ne font l'objet 
d'aucune classification. Ces prescriptions portent sur les temps et les modes à enseigner, ainsi que sur les formes non finies du verbe et les auxiliaires, tels qu'on peut les trouver dans une grammaire scolaire, mais avec certaines restrictions.

Ainsi, dans le Français fondamental 1er degré, on limite l'enseignement des temps au présent, à l'imparfait et au passé composé, au détriment du passé simple et du passé antérieur, tandis qu'on recommande l'introduction « progressive » du plus-que-parfait et du futur antérieur. De façon analogue, il est préconisé d'enseigner le subjonctif de façon limitée et uniquement dans des tournures telles qu 'avant que, pour que, avoir peur que, il faut que, vouloir que etc. (cf. la section 1.3. supra ). L'Élaboration du Français fondamental note en effet que la moitié des subjonctifs apparaissent avec le verbe vouloir.

En relation avec l'idée qu'il existe des " connaissances passives " à acquérir, on enseigne à « reconnaître » le passé antérieur et la forme je puis.

Par ailleurs, alors que les auxiliaires avoir et être sont enseignés avec le participe passé, lequel n'apparaît que dans les temps composés du verbe, la règle de l'accord du participe passé dans le cas de avoir est écartée du 1er degré et intégrée au 2ème degré.

Aucune explication n'est donnée sur le temps et sur l'harmonisation des temps ; aucune indication n'est délivrée sur le mode de progression à adopter pour introduire les autres temps composés mentionnés ci-dessus. Les critères ayant prévalu pour les choix de temps à enseigner sont fondés sur la différence faite entre l'utilisation des temps à l'oral (français parlé) et à l'écrit chez des natifs ; ceci se trouve confirmé par la suite dans le Français fondamental 2ème degré.

\subsubsection{Le Français fondamental 2ème degré}

16 À la suite du 1er degré, le 2ème degré introduit de nouveaux éléments. Le présent, l'imparfait et le passé composé sont toujours enseignés. Des prescriptions sont données pour les auxiliaires :

-L'usage de avoir et être est étendu à tous les verbes de la liste. On enseigne : « il a couru », «il est arrivé », « il s'est blessé ». Aucune explication n'est donnée sur les différents emplois de ces auxiliaires, ni sur la transitivité et l'intransitivité des verbes. On remarque l'absence de la forme « il a blessé x » qui pourrait faire pendant à « il s'est blessé ». Cette absence s'explique du fait que la syntaxe du verbe n'est pas l'objet de la grammaire du Français fondamental, à deux domaines près, l'interrogative et la subordonnée complétive.

- La règle d'accord du participe passé suivant l'auxiliaire avoir est enseignée.

Le passé simple est introduit mais n'est enseigné qu'aux troisièmes personnes du singulier et du pluriel, alors qu'on apprend à manier le passé antérieur au préalable reconnu dans le 1er degré. Malgré la déclaration d'intention des auteurs, à savoir l'absence de doctrine, on reconnaît l'influence d'une linguistique énonciative. Il s'agit sans doute de celle d'E. Benveniste (1966: 239) qui opposera bien après la publication du Français fondamental, "temps de l'histoire " à " temps du discours ", et du même coup la différence entre l'oral et l'écrit dans l'usage des temps.

\subsection{Synthèse}

La partie grammaticale du Français fondamental 1er et 2ème degrés est constituée de listes de prescriptions restrictives. Elles influent sur l'ordre d'introduction des 
contenus grammaticaux créant une relation de "suite» entre le premier et le deuxième degrés, censés favoriser les « connaissances actives " puis les « connaissances passives ».

Les verbes font partie de la liste générale proposée, par ordre alphabétique, de mots non grammaticaux, sans qu'ils soient abordés sous l'angle syntaxique à quelques exceptions près. Ainsi, les auteurs ont été sensibles aux contextes où des locutions conjonctives introduisent le subjonctif, comme le montre le chapitre de L'élaboration du Français fondamental consacré à la grammaire (Gougenheim et al, 1956 / 1964). De même, ils ont noté la douzaine de verbes d'une fréquence élevée régissant des subordonnées complétives, comme vouloir que, croire que, savoir que, penser que etc. Si l'accent est mis avant tout sur les temps du verbe, ils ne servent cependant pas de critère de classification. La morphologie verbale et la conjugaison ne sont ni abordées, ni présentées intégralement comme dans les manuels scolaires, ni comme elles l'ont été plus tardivement, sous forme de bases (Martinet 1974 ; Dubois 1967).

Le point de vue adopté dans le Français fondamental reste celui d'un compromis, car l'objectif n'est pas de formuler une doctrine grammaticale. Les listes de prescriptions esquissent une grammaire de l'oral ; on peut citer, sans viser à l'exhaustivité, la réduction du paradigme verbal à apprendre, la limitation drastique de celui des pronoms relatifs, les choix en matière de construction interrogative, lesquels vont dans ce sens. Quant à l'ouvrage de Le Goffic et de Combe Mc Bride (1975) qui lui succède, il participe d'un mouvement d'élaboration des contenus dans le cadre défini par le Français fondamental, lequel prévaut, selon R. Vives (1989), jusqu'en 1972-1974. Néanmoins, Les constructions fondamentales du français diffèrent des prescriptions grammaticales du Français fondamental et adoptent un point de vue théorique tranché pour traiter les mêmes données lexicales.

\section{Les Constructions fondamentales du français: inventaire de constructions et dictionnaire syntaxique}

\subsection{L'objet des Constructions fondamentales du français}

21 Cet ouvrage propose un cadre syntaxique pour les listes du Français fondamental 1er degré. Seuls les verbes (350 unités) et les adjectifs (150 unités) sont retenus en tant qu'« inducteurs» de phrases où ils jouent le rôle de " pivots » (CFDF : 15), ce qu'on peut voir dans les exemples suivants :

(1) Pierre donne un livre à Marie.

(2) Pierre est sûr que Marie viendra.

En (1) le verbe sélectionne deux compléments dont le deuxième est introduit par à, alors qu'en (2) la structure en N + être + Adj. + que + phrase à l'indicatif, n'est possible qu'avec certains adjectifs. Cet aspect est totalement absent du Français fondamental dont les objectifs diffèrent de ceux des Constructions fondamentales du français. En effet, ces dernières veulent favoriser l'acquisition des structures syntaxiques chez les apprenants en commençant par celles du verbe et de l'adjectif. 


\subsection{Les cadres théorique et méthodologique} verbes et des adjectifs listés dans le Français fondamental 1er degré. Les critères reposent sur l'intuition linguistique des auteurs, natifs de la langue, et sur les jugements d'acceptabilité d'autres natifs. Bien que la nomenclature soit celle de la grammaire scolaire (verbes pronominaux, impersonnels etc.), l'angle d'analyse retenu est celui de la linguistique distributionnelle et transformationnelle, dans le cadre de la théorie de M. Gross. Plusieurs réalisations (structures de surface) sont postulées pour une même structure profonde (Gross 1968, Harris 1970, Gross et Stefanini 1971). C'est le cas cidessous (cf. CFDF : 19) où il existe un rapport de transformation entre (3) et (4) :

J'espère que je viendrai.

J'espère venir.

(4) est une transformation de (3) par " réduction » de la complétive, ce sur quoi nous reviendrons en 2.4.1. Ce type d'analyse est totalement absent du Français fondamental 1er et 2ème degrés où certes les constructions des verbes apparaissent, mais comme des tournures énumérées, sans qu'on établisse de lien entre elles. Au contraire, la démarche des Constructions fondamentales du français permet de visualiser plusieurs formes disponibles dans la langue, pour signifier le même état de choses ou le même événement.

\subsection{Les aspects complémentaires des Constructions fondamentales du français}

Selon ses auteurs, l'ouvrage peut être consulté comme un dictionnaire centré sur les emplois des verbes et des adjectifs (et non sur leur sens), mais aussi comme une grammaire qui présente diverses structures de phrases possibles avec les unités listées dans le Français fondamental 1er degré.

Pour ce qui concerne le premier aspect, cela renseigne par exemple le non-natif qui ne peut décider de l'emploi d'une préposition après un verbe (ou un adjectif). Certains verbes peuvent se combiner avec plusieurs prépositions, ce qui représente une difficulté supplémentaire (croire en / croire à ; parler de / parler à etc.). On se reporte alors à l'index des verbes et des adjectifs. Quant au deuxième aspect, on peut rechercher quels verbes se construisent avec une complétive, un subjonctif, admettent un attribut de l'objet, etc., et on consulte l'index des constructions et le plan détaillé (pp. 39, 88, 111, 139).

Les auteurs précisent que leur ouvrage ne peut être considéré comme une " grammaire fondamentale ", car une telle grammaire ne peut être réalisée. La grammaire du Français fondamental est constitutive du choix délibéré des auteurs d'organiser essentiellement l'enseignement des constructions verbales et adjectivales.

Pour ces mêmes raisons l'idée de compter les fréquences de structures pour établir leur liste, comme cela a été fait pour le Français fondamental est écartée. Plusieurs arguments sont avancés par Le Goffic et Combe Mc Bride (1975 : 17) ; nous ne les évoquerons pas ici. L'un d'eux s'appuie sur la difficulté à compter des unités que l'on n'a pas au préalable définies et délimitées. Le Français fondamental 1er degré pèche effectivement par l'absence de définition du mot et de l'unité lexicale, comme nous l'avons fait remarquer supra en 1.2. Le comptage de "structures » est sans conteste encore plus

Documents pour l'histoire du français langue étrangère ou seconde, 36 | 2006 
périlleux que celui de "mots ». En effet, pour pouvoir dénombrer des "structures ", il faut les avoir définies en amont. Les auteurs montrent la difficulté à décomposer une phrase en s'appuyant sur des exemples. De plus, ils justifient leur hostilité aux méthodes de comptage et de fréquence en faisant référence aux travaux de J. C. Corbeil ${ }^{3}$ (1968) dans lesquels l'utilité des apports statistiques n'est pas prouvée, et ce, selon l'aveu même de l'auteur. La seule intuition native, sans le recours à des statistiques, laisse supposer que dans un texte écrit (non littéraire) la structure la plus fréquente sera celle de "Sujet Verbe Objet ». Ceci suffit pour affirmer que c'est la construction la plus usuelle

\subsection{Le traitement du verbe}

\subsubsection{Les « constructions nominales » vs « opératrices »}

$$
\text { page est consacrée aux « verbes symétriques » (Je colle le timbre vs le timbre colle), }
$$
notion empruntée à J. Dubois (1967).

\subsubsection{Les temps verbaux}

Les temps sont abordés d'un point de vue syntaxique ce qui élimine certains aspects de la grammaire scolaire conservés dans le Français fondamental. Nous nous bornerons à commenter un exemple. Le choix de l'indicatif ou du subjonctif après que dépend du verbe mais rien n'est dit sur le sémantisme de son procès, ni sur celui des modes indicatif vs subjonctif (vouloir que + SUBJ vs s'apercevoir que + IND). En revanche, 
lorsque les deux modes sont possibles pour un verbe donné + que, les auteurs proposent une explication sémantique sous forme de paraphrase (ce que nous avons noté en caractères gras) :

(7) Maintenant je comprends que je me suis trompé. =je me rends compte que je me suis trompé.

(8) Maintenant je comprends que je me sois trompé. =je comprends pourquoi je me suis trompé.

Ces paraphrases ne suffisent pas à justifier l'alternance de modes après que auprès d'un non-natif du français, lequel pourrait très bien accepter *je me rends compte que je me sois trompé, du fait qu'il n'a pas d'intuition sur la langue qu'il apprend. De plus il lui faut construire des paraphrases (en recourant à des para synonymes), et accéder aux différences de sens entre les deux phrases obtenues pour inférer des critères sélectifs (choix de l'infinitif vs de la complétive, ou les deux).

\subsection{Synthèse}

L'ouvrage de Le Goffic et Combe Mc Bride apporte des compléments aux propositions du Français fondamental en s'intéressant à la construction de la phrase. Cependant, la relation de continuité entre les deux ouvrages est compromise par le point de vue analytique des Constructions fondamentales du français, directement inspiré du modèle de Gross (cf. supra, 2.2.). Dans cette optique, l'acquisition de la syntaxe de constituants majeurs (les verbes et les adjectifs) en français L2, requiert qu'on maitrise les mécanismes transformationnels. Or, on sait qu'ils ne sont pas le reflet d'opérations mentales projetées par le locuteur ; ce dernier construit et produit des phrases sans passer par des étapes de transformations dont le traitement serait coûteux. D'ailleurs, de tels mécanismes ne sont pas directement disponibles chez le natif qui ne les utilise qu'une fois formé à la théorie syntaxique de référence. Bref, l'arsenal transformationnel ne délivre pas d'explications qui facilitent forcément l'acquisition de la syntaxe de la langue. Dans le contexte de l'ouvrage de Le Goffic et de Combe Mc Bride, il constitue plutôt un outil qui permet de décrire la syntaxe du verbe et de l'adjectif; de ce côté-là il inculquerait plutôt à l'apprenant un savoir métalinguistique en le formant à un modèle d'analyse syntaxique. D'un autre côté, depuis la tentative de ces deux auteurs, les théories syntaxiques ont évolué. Ainsi, en reprenant les exemples (3) et (4), on parlera plus volontiers de formes finie vs non finie ou de complétives tensée vs non tensée ; on traitera les deux constructions comme des complétives, l'une introduite par que, l'autre non tensée précédée ou non d'un complémenteur, le tout sans passer par des transformations.

\section{Conclusion}

Au terme de cette étude comparative, il apparaît que le Français fondamental, qu'on a pu juger avare d'explications grammaticales car ne s'appuyant sur aucune théorie, n'a pas vieilli, même s'il n'a pas innové sur le plan de l'analyse syntaxique. Il constitue même un matériel d'avant-garde dans le contexte de son époque ${ }^{6}$. Il faut y lire en creux les recommandations de la Commission du Français élémentaire en matière de grammaire, comme on le fait pour les auteurs de remarques du dix-septième siècle, afin d'en dégager une grammaire de l'oral. Il apparaît en effet que le Français fondamental conserve une certaine actualité linguistique, voire didactique, quand on connaît 
l'intérêt de ces dernières années pour la grammaire de l'oral, les travaux de didacticiens menés sur l'interaction et la constitution de corpus à partir d'enregistrements.

Les prolongements que propose l'ouvrage de Le Goffic et de Combe Mc Bride (1975), lesquels se sont préoccupés des propriétés distributionnelles et syntaxiques des verbes et des adjectifs du Français fondamental, ne semblent pas avoir exercé une influence directe dans le champ du FLE. On n'en trouve nulle référence dans la plupart des travaux produits sur la grammaire. D'ailleurs, le Niveau B2 pour le français (Beacco, Bouquet, Porquier 2004) emploie à dessein, une version très épurée de grammaire syntagmatique. L'apport grammatical et la solidité des analyses de Le Goffic et de Combe Mc Bride ne sont certes pas en cause. Leur travail n'a pas eu de suite pour des raisons qui tiennent sans doute à la nature même des analyses proposées, mais aussi à la conjoncture didactique. L'on peut y voir l'indice d'une résistance à l'emploi d'une démarche encore trop marquée par la linguistique transformationnelle. D'un autre côté, au moment où est publié l'ouvrage de Le Goffic et de Combe Mc Bride, la tendance en didactique du français langue étrangère est celle d'un développement des approches onomasiologiques et d'une prise de distance par rapport à la linguistique appliquée.

L'évolution que nous venons d'esquisser et de discuter en comparant le traitement du verbe dans le Français fondamental et les Constructions fondamentales du Français est à mettre en relation avec la question de l'enseignement de la grammaire en didactique du FLE. Comme Henri Besse (1994) a su le montrer, y co-existent plusieurs visions de la règle grammaticale, d'où différents modes d'enseignement de la grammaire. Le Français fondamental formule des prescriptions qui ont modelé la progression des manuels et des méthodes des années 70, tandis que les structures mises à jour par les Constructions fondamentales du français appellent une lecture didactique plus complexe, qui ne pouvait avoir lieu à une période de rejet des analyses linguistiques en didactique par les didacticiens. Tout ceci explique que la continuité entre ces deux ouvrages, se soit soldée par l'oubli du dernier, trop marqué théoriquement. On ne reconnaît d'ailleurs pas son empreinte, ni sur le Niveau Seuil (Coste et al. 1976), ni sur le Niveau B2 pour le français (Beacco, Bouquet, Porquier 2004).

On sait que l'avènement de la démarche notionnelle en matière de grammaire après 1976, a conduit à un mouvement de flux et de reflux dans son enseignement. Si la grammaire notionnelle du Niveau Seuil appelle à un travail de conceptualisation sur une base sémantique, le Niveau B2 pour le français atteste dans la conception de sa grammaire d'un retour vers une analyse distributionnaliste et d'une nomenclature grammaticale teintée de syntaxe structurale. On est ainsi passé d'une grammaire notionnelle à une grammaire syntagmatique, de l'abandon d'une terminologie grammaticale scolaire à son retour avec quelques aménagements, provenant de la linguistique structurale. Nous aurons l'occasion d'y revenir lors d'un prochain travail. 


\section{BIBLIOGRAPHIE}

BEACCO, J-C, BOUQUET, S., PORQUIER, R. (2004), Niveau B2 pour le français/ un référentiel, Paris : Didier.

BENVENISTE, E. (1966), Problèmes de linguistique générale, Paris :Seuil.

BESSE, H. et GALISSON, R. (1980), Polémique en didactique. Du renouveau en question, Paris : CLE International.

BESSE, H. (1994), «L'enseignement de la "grammaire" du français langue seconde ou étrangère de 1968 à 1988 ", Vingt ans dans l'évolution de la didactique des langues (1968-1988), Coste D. (éd.), Paris: Hatier / Didier, pp. 55-69.

CHEVALIER, J-C. (1984), « Linguistique appliquée et linguistique tout court », Vingt ans dans l'évolution de la didactique des langues (1968-1988), CosteD. (éd.), Paris : Hatier / Didier, pp. 119-126.

CORBEIL, J-C. (1968), Les structures syntaxiques du français moderne, Paris : Klincksieck.

COSTE, D. et al. (1976), Un niveau seuil, Strasbourg, Conseil de l'Europe.

COSTE, D. (1989), «La grammaire entre linguistique et didactique», Grammaire et français langue étrangère, JAUSSAUD A.-M, PETRISSANS J. (éds.), Grenoble : A.N.E.F.L.E, pp. 3-14.

DUBOIS, J. (1967), Grammaire structurale du français : le verbe, Paris : Larousse.

GOUGENHEIM, G., MlCHEA, M., RlVENC, P., SAUVAGEOT, A. (1956/1964), L'élaboration du Français fondamental (1er degré). Étude sur l'élaboration d'un vocabulaire et d'une grammaire de base, Paris : Didier.

GROSS, M. (1968), Grammaire transformationnelle du français :syntaxe du verbe, Paris : Larousse.

GROSS, M., STEFANINI, J. (1971), « Syntaxe transformationnelle du français », Langue Française, 11, Paris : Larousse.

HARRIS, Z. (1970), Papers on Syntax, Dordrecht : Reidel.

LE GOFFIC, P., COMBE MC BRIDE, N. (1975), Les constructions fondamentales du français, Paris : Hachette et Larousse.

MINISTÈRE DE I'ÉDUCATION Nationale, ENS de Saint Cloud, (1972), Le Français fondamental 1er Degré, 2ème degré, Institut National de Recherche et de Documentation Pédagogique.

MARTINET, A. (1974), « De l'économie des formes du verbe en français parlé », Le français sans fard, MARTINET A (éd.), Paris : PUF, pp.91-120.

VIVES, R. (1989), « D'hier à demain : la grammaire dans tous ses états », Le Français dans le Monde. Recherches et Applications, pp. 92-102.

\section{NOTES}

1. Brochure de l'Institut national de recherche et documentation pédagogique, $1972: 1-2$

2. Le Français fondamental 2eme degré, brochure 212 de l'INRP, p. 47.

3. Cette étude définit le concept de «structure fondamentale » en observant des arrangements / genres discursifs et leur fréquence 
4. Chez M. Gross les prépositions à, de précédant les infinitifs, ainsi que la conjonction que sont traitées comme des opérateurs dans les transformations complétives et infinitives

5. Ces exemples sont proposés par les auteurs de l'article.

6. D'où les nombreuses critiques et polémiques qu'il a suscitées à l'époque de sa création.

\section{AUTEURS}

DOMINIQUE KLINGER

Université Paris III

\section{GEORGES DANIEL VÉRONIQUE}

Université de Provence61-75 